\title{
Compactação do solo e intervalo hídrico ótimo na produtividade de arroz de sequeiro
}

Amauri Nelson Beutler ${ }^{(1)}$, José Frederico Centurion ${ }^{(1)}$, Alvaro Pires da Silva( ${ }^{(2)}$, Cassiano Garcia Roque ${ }^{(1)}$ e Marcos Vieira Ferraz ${ }^{(1)}$ (1) Universidade Estadual Paulista, Fac. de Ciências Agrárias e Veterinárias, Dep. de Solos e Adubos, Via de Acesso Prof. Paulo Donato
Castellane s/no, CEP 14870-000 Jaboticabal, SP. E-mail: amaurib@yahoo.com.br, jfcentur@fcav.unesp.br (2)Escola Superior de Agricultura Luiz de Queiroz, Dep. de Ciência do Solo, Caixa Postal 09, CEP 13418-900 Piracicaba, SP.

Resumo - O objetivo deste trabalho foi determinar o intervalo hídrico ótimo e sua relação com a produtividade de grãos de arroz de sequeiro em um Latossolo Vermelho de textura média. Os tratamentos foram $0,1,2,4$ e 6 passadas de um trator, lado a lado, na superfície do solo, com quatro repetições. O delineamento experimental foi inteiramente casualizado. Foram coletadas amostras indeformadas de solo nas camadas de $0,02-0,05,0,07-0,10$ e $0,15-0,18$ m para determinação da resistência do solo à penetração, densidade do solo, intervalo hídrico ótimo, densidade do solo crítica e da curva de retenção de água no solo. Em parcelas de 3,6 $\mathrm{m}^{2}$ foi semeado arroz de sequeiro (cv. Caiapó) e a sua produtividade foi avaliada em parcelas úteis de $1,80 \mathrm{~m}^{2}$. O intervalo hídrico ótimo foi reduzido a partir da densidade do solo de $1,30 \mathrm{Mg} \mathrm{m}^{-3}$ pela resistência do solo à penetração. A partir do valor de 1,82 MPa ocorreu decréscimo da produtividade de arroz. A densidade do solo crítica ao desenvolvimento radicular no intervalo hídrico ótimo foi de $1,63 \mathrm{Mg} \mathrm{m}^{-3}$, equivalente a $1,62 \mathrm{Mg} \mathrm{m}^{-3}$ que foi limitante na produtividade de arroz. O monitoramento da compactação do solo é necessário para prevenir perdas na produtividade de arroz de sequeiro.

Termos para indexação: Oryza sativa, resistência do solo à penetração, água no solo, qualidade física do solo.

\section{Soil compaction and least limiting water range in dryland rice yield}

Abstract - The objective of this work was to determine the least limiting water range and its relation with dryland rice yield in a medium texture Oxisol. The treatments were $0,1,2,4$ and 6 passed of a tractor, side by side, on soil surface. The experimental design was a completely randomized, with four repetitions. Indeformed soil samples were collected in the layers $0.02-0.05,0.07-0.10$ and $0.15-0.18 \mathrm{~m}$ for determination of soil resistance to penetration, bulk density, least limiting water range, critical bulk density and soil water retention curve. In $3.6 \mathrm{~m}^{2}$ plots dryland rice (cv. Caiapó) was sown and its yield in useful parcels of $1.80 \mathrm{~m}^{2}$ was evaluated. The least limiting water range was reduced from bulk density of $1.30 \mathrm{Mg} \mathrm{m}^{-3}$ by soil resistance to penetration. From $1.82 \mathrm{MPa}$ on decrease of rice yield occurred. Critical bulk density to root development in the least limiting water range was $1.63 \mathrm{Mg} \mathrm{m}^{-3}$, equivalent to $1.62 \mathrm{Mg} \mathrm{m}^{-3}$ of bulk density, that is critical to rice yield. The monitoring of soil compaction is necessary to prevent losses in dryland rice yield.

Index terms: Oryza sativa, resistance to penetration, soil water, soil physical quality.

\section{Introdução}

A cultura de arroz de sequeiro (arroz de terras altas) tem sido utilizada, principalmente na região dos cerrados, como alternativa na rotação de culturas em sistemas de plantio direto (SPD) (Moura Neto, 2001) No entanto, no SPD ocorre impedimento mecânico ao crescimento radicular, em razão da maior compactação do solo na camada de 0,075-0,17 m (Silva et al., 2000), quando comparado aos sistemas de manejo convencional com revolvimento anual do solo. Em virtude da recente utilização dessa cultura neste sistema de manejo são escassos os estudos definindo níveis de atributos físicos indicadores da compactação do solo (Moura Neto, 2001).
Além disso, verifica-se dificuldade de avaliar a compactação do solo originada do excessivo tráfego de máquinas em condições de solo muito úmido. Ralisch \& Tavares Filho (2002) afirmam que a compactação provoca significativas reduções na produtividade e acréscimos nos custos de produção das culturas anuais.

Na compactação do solo, há aumento de massa por unidade de volume, acarretando maior densidade do solo e resistência do solo à penetração, com redução linear da porosidade total e da macroporosidade (Stone et al., 2002). As conseqüências da compactação se manifestam no solo e na planta. No solo, ocorre a presença de zonas endurecidas, empoçamento de água, erosão hídrica, poluição e assoreamento dos mananciais de água. Na planta, ocorre redução da penetração das raízes por 
excessiva resistência do solo à penetração, deficiência de oxigênio, menor desenvolvimento vegetativo da parte aérea e redução significativa da produtividade (Hakansson \& Voorhees, 1998).

O atributo físico adotado como indicativo da compactação do solo tem sido a resistência do solo à penetração (Imhoff et al., 2000; Stone et al., 2002), por apresentar relações diretas com o crescimento das plantas (Hoad et al., 2001) e por ser mais eficiente na identificação de estados de compactação comparada à densidade do solo (Silva, 2003). A resistência do solo à penetração é influenciada pelo conteúdo de água, textura e pela condição estrutural do solo (Tardieu, 1994), fatores que dificultam a obtenção de valores críticos às plantas.

Dessa forma, a resistência à penetração geralmente é determinada quando o conteúdo de água no solo equivale à capacidade de campo (Smith et al., 1997), para que seja possível estabelecer valores limitantes e fazer inferências em relação ao valor crítico de 2,0 MPa ao crescimento radicular das plantas (Silva et al., 1994; Tormena et al., 1998). Imhoff et al. (2000) adotaram o valor de 2,5 MPa como limitante na pastagem com capim-elefante (Pennisetum purpureum) na determinação da curva de resistência do solo à penetração em razão do conteúdo de água do solo, no controle da qualidade física do solo. Mielniczuk et al. (1985), em casa de vegetação, verificaram redução na massa de matéria seca da parte aérea de aveia e trigo a partir da resistência do solo à penetração de 2,3 MPa em Latossolo Roxo. Merotto \& Mundstock (1999) verificaram menor produtividade de matéria seca de trigo a partir da resistência do solo à penetração de 2,0 MPa em Podzólico Vermelho-Escuro, em casa de vegetação.

$\mathrm{Na}$ determinação da resistência do solo à penetração, são utilizados diversos penetrômetros, o que tem dificultado a interpretação dos resultados. Assim, Herrick \& Jones (2002) recomendam a utilização de penetrômetros estáticos com velocidade constante de penetração tanto por sua maior precisão como também para padronizar as determinações.

Além da resistência à penetração, surgiu recentemente o intervalo hídrico ótimo, introduzido no Brasil por Tormena et al. (1998), no qual as limitações ao crescimento das raízes por causa da disponibilidade de água, aeração e resistência do solo à penetração são mínimas. O limite superior é o conteúdo de água na capacidade de campo na tensão de 0,01 MPa (Reichardt, 1988) ou o conteúdo de água no qual a porosidade de aeração é de $10 \%$; e o limite inferior é o conteúdo de água no ponto de murcha permanente na tensão de 1,5 $\mathrm{MPa}$ ou a resistência do solo à penetração de 2,0 $\mathrm{MPa}$ (Silva et al., 1994).

Silva (2003), estudando o intervalo hídrico ótimo na cultura do feijão, em Latossolo Vermelho, verificou que, no preparo reduzido do solo, a cultura permaneceu três dias a mais no intervalo hídrico ótimo ao desenvolvimento da planta comparado ao preparo convencional e plantio direto, contudo não houve diferença na produtividade da cultura.

O objetivo deste trabalho foi determinar o intervalo hídrico ótimo e sua relação com a produtividade de grãos de arroz de sequeiro em um Latossolo Vermelho de textura média.

\section{Material e Métodos}

O estudo foi realizado na FCAV/Unesp, Jaboticabal, SP (21 15' 29" S, 48 16' 53" W; 607 m de altitude). O clima é do tipo Cwa, segundo a classificação de Köppen. As precipitações pluviais diárias durante o ciclo da cultura do arroz de sequeiro são apresentadas na Figura 1.

Utilizou-se um Latossolo Vermelho distrófico, típico, textura média, A moderado, caulinítico hipoférrico (LVd), cuja composição granulométrica, determinada pelo método da pipeta (Embrapa, 1997) foi: argila, $271 \mathrm{~g} \mathrm{~kg}^{-1}$; silte, $42 \mathrm{~g} \mathrm{~kg}^{-1}$ e areia, $687 \mathrm{~g} \mathrm{~kg}^{-1}$, na camada de $0,0-0,20 \mathrm{~m}$.

A área experimental recebeu calcário e havia sido cultivada com soja no ano anterior. Antes da instalação do experimento, foi realizada uma escarificação do solo até $0,30 \mathrm{~m}$ de profundidade, seguida de uma gradagem. Dois dias após intensa precipitação pluvial, o solo atingiu conteúdo de água próximo à capacidade de campo

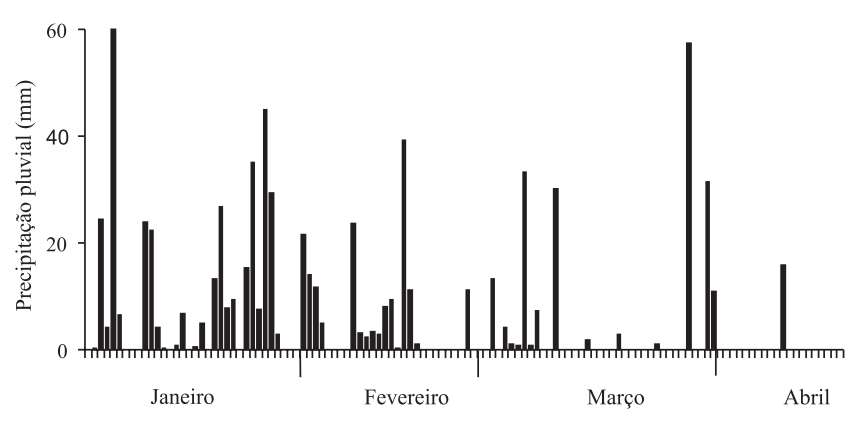

Figura 1. Precipitação pluvial diária durante o ciclo da cultura de arroz de sequeiro. No mês de maio ocorreu a precipitação de $66,7 \mathrm{~mm}$, nos dias quatro e cinco. 
(0,01 MPa), quando foi realizada a sua compactação por meio da passagem lado a lado de um trator de $11 \mathrm{Mg}$ com os quatro pneus de mesma largura $(0,40 \mathrm{~m})$ e pressão interna, perfazendo toda a superfície do solo. Os tratamentos constituíram-se de $0,1,2,4$ e 6 passadas de trator, cobrindo toda a área.

$\mathrm{O}$ delineamento experimental foi inteiramente casualizado com cinco tratamentos e quatro repetições, com parcelas medindo 1,60 m de comprimento e 2,25 m de largura e área útil de $1,8 \mathrm{~m}^{2}$.

No início de janeiro de 2003, foi semeado arroz (cv. Caiapó) na profundidade de $0,05 \mathrm{~m}$ e espaçamento de 0,45 m entre linhas e o desbaste foi realizado 10 dias após a germinação, deixando 80 plantas por metro linear. Em maio foi avaliada a produtividade de grãos de arroz.

A análise química do solo foi realizada conforme Raij et al. (1987). Aplicaram-se $0,25 \mathrm{Mg} \mathrm{ha}^{-1}$ da fórmula 4-20-20 para produtividade esperada de 2,50-4,00 $\mathrm{Mg} \mathrm{ha}^{-1}$, segundo Raij et al. (1996). Aplicou, em cobertura, 30 dias após a semeadura, $0,14 \mathrm{Mg} \mathrm{ha}^{-1}$ de uréia.

$\mathrm{Na}$ determinação do intervalo hídrico ótimo (IHO), foram coletadas duas repetições de seis amostras indeformadas com cilindros de $53,16 \times 10^{-6} \mathrm{~m}^{3}$, nas camadas de $0,02-0,05,0,07-0,10$ e $0,15-0,18 \mathrm{~m}$, totalizando 36 amostras por tratamento, as quais foram saturadas e submetidas às pressões de $0,006,0,01,0,033,0,06,0,1 \mathrm{e}$ $0,3 \mathrm{MPa}$, com o aparelho de placas porosas de Richards (Embrapa, 1997). Ao atingir o equilíbrio, foi realizada a pesagem das amostras e duas determinações de resistência do solo à penetração (RP), por amostra, na camada de $0,01-0,02 \mathrm{~m}$, perfazendo 200 leituras, as quais foram utilizadas na obtenção da RP média.

A RP foi determinada com o penetrômetro eletrônico estático com velocidade constante de penetração de $0,01 \mathrm{~m} \mathrm{~min}^{-1}$ e cone com área de $3,14 \times 10^{-6} \mathrm{~m}^{2}$, que realiza 100 leituras por centímetro. Este penetrômetro é equipado com atuador linear e célula de carga de $20 \mathrm{~kg}$ acoplada a um microcomputador, conforme descrito por Tormena et al. (1998). Os valores obtidos $\left(\mathrm{kgf} \mathrm{cm}^{-2}\right)$ foram multiplicados pelo fator 0,098 para transformação em MPa. Na Tabela 1 são apresentados os valores de RP e densidade do solo (Ds).

Nestas amostras foram determinados o conteúdo de água retida em cada tensão (Embrapa, 1997) e a Ds (Embrapa, 1997). As curvas de retenção de água no solo foram ajustadas pelo modelo proposto por Genuchten (1980), a partir do qual foi obtido o conteúdo de água retida na capacidade de campo (tensão de
0,01 MPa) e no ponto de murcha permanente (tensão de $1,5 \mathrm{MPa}$ ).

Os valores de RP foram ajustados em relação ao conteúdo de água volumétrica e a Ds, utilizando o modelo não-linear proposto por Busscher (1990), na sua forma log-transformada, descrito a seguir:

$\ln R P=\ln a+b \ln \theta+\ln D s$

em que RP é a resistência do solo à penetração $(\mathrm{MPa})$, $\theta$ é o conteúdo de água volumétrico $\left(\mathrm{m}^{3} \mathrm{~m}^{-3}\right)$, Ds é a densidade do solo $\left(\mathrm{Mg} \mathrm{m}^{-3}\right)$ e $\mathrm{a}, \mathrm{b}$, c são coeficientes obtidos por meio do ajuste do modelo.

O IHO foi determinado para cada amostra conforme Silva et al. (1994), formando em conjunto o conteúdo de água ótimo em função da densidade do solo.

Os resultados foram submetidos à análise de variância e as médias de produtividade de grãos de arroz foram comparadas pelo teste de Tukey a $5 \%$ de probabilidade.

\section{Resultados e Discussão}

Com a finalidade de aumentar a precisão do intervalo hídrico ótimo no monitoramento da qualidade do solo, foi determinado o valor de RP a partir do qual a produtividade do arroz decresce (Tabela 2). Como a regressão quadrática entre a RP e a produtividade de arroz não foi significativa, realizou-se o teste de médias para definir o valor a partir do qual a produtividade foi reduzi-

Tabela 1. Resistência do solo à penetração e densidade do solo nos diferentes tratamentos e camadas no Latossolo Vermelho distrófico textura média.

\begin{tabular}{|c|c|c|c|c|c|}
\hline \multirow[t]{2}{*}{$\overline{\text { Camada }(\mathrm{m})}$} & \multicolumn{5}{|c|}{ Tratamentos $^{(1)}$} \\
\hline & $\mathrm{T}_{0}$ & $\mathrm{~T}_{1}$ & $\mathrm{~T}_{2}$ & $\mathrm{~T}_{4}$ & $\mathrm{~T}_{6}$ \\
\hline & \multicolumn{5}{|c|}{ Resistência do solo à penetração $(\mathrm{MPa})^{(2)}$} \\
\hline $0,02-0,05$ & 0,21 & 1,00 & 1,92 & 3,58 & 4,57 \\
\hline $0,07-0,10$ & 0,32 & 2,38 & 2,63 & 4,40 & 4,10 \\
\hline $0,15-0,18$ & 0,65 & 2,07 & 3,65 & 3,64 & 4,07 \\
\hline $0,25-0,28$ & $-^{(3)}$ & - & - & . & - \\
\hline \multirow[t]{2}{*}{ Média $(0,0-0,20)$} & 0,39 & 1,82 & 2,40 & 3,87 & 4,25 \\
\hline & \multicolumn{5}{|c|}{ Densidade do solo $\left(\mathrm{Mg} \mathrm{m}^{-3}\right)$} \\
\hline $0,02-0,05$ & 1,19 & 1,54 & 1,70 & 1,74 & 1,80 \\
\hline $0,07-0,10$ & 1,31 & 1,68 & 1,76 & 1,82 & 1,81 \\
\hline $0,15-0,18$ & 1,46 & 1,64 & 1,74 & 1,77 & 1,78 \\
\hline $0,25-0,28$ & 1,51 & 1,53 & 1,61 & 1,66 & 1,66 \\
\hline Média $(0,0-0,20)$ & 1,32 & 1,62 & 1,73 & 1,78 & 1,80 \\
\hline
\end{tabular}

Pesq. agropec. bras., Brasília, v.39, n.6, p.575-580, jun. 2004 
da, o que ocorreu na RP de 1,82 MPa, valor próximo ao de 2,00 $\mathrm{MPa}$, adotado como limitante ao crescimento radicular (Tormena et al., 1998) e da parte aérea de trigo (Merotto \& Mundstock, 1999). Assim, utilizou-se o valor de 2,00 MPa no intervalo hídrico ótimo.

Em casa de vegetação, Rosolem et al. (1999) verificaram que a RP de $1,30 \mathrm{MPa}$, determinada com o penetrômetro de bolso, em Latossolo Vermelho e Latossolo Roxo, reduziu o crescimento das raízes seminais adventícias do milho. Tavares Filho et al. (2001) verificaram que a RP de 3,50 MPa, determinada com o penetrômetro de impacto, em Latossolo Roxo, no campo, não restringiu o desenvolvimento radicular do milho.

Os diferentes valores críticos de RP entre arroz milho podem ser explicados, em parte, pelos penetrômetros utilizados nas determinações. Segundo Beutler et al. (2002), o penetrômetro de impacto determina valores superiores ao penetrômetro estático e essas diferenças aumentam com a compactação e o teor de argila, evidenciando a necessidade de padronização de penetrômetros, conforme mencionado por Herrick \& Jones (2002).

A Ds foi crítica à produtividade de arroz a partir de $1,62 \mathrm{Mg} \mathrm{m}^{-3}$ (Tabela 2), valor superior a $1,55 \mathrm{Mg} \mathrm{m}^{-3}$, considerado crítico por Camargo \& Alleoni (1997) para solos franco-argilosos. Guimarães \& Moreira (2001) verificaram no campo redução na produtividade de matéria seca da parte aérea de arroz (cv. Caiapó e Cirad L-141) a partir da Ds de $1,40 \mathrm{Mg} \mathrm{m}^{-3}$ em Latossolo Vermelho-Escuro de textura média. Esta redução da produtividade de matéria seca da parte aérea da mesma cultivar de arroz em níveis inferiores de Ds, em solo

Tabela 2. Produtividade relativa (\%) de grãos de arroz de sequeiro em função da resistência do solo à penetração e densidade do solo no Latossolo Vermelho distrófico.

\begin{tabular}{cccc}
\hline Tratamentos $^{(1)}$ & $\begin{array}{c}\text { Resistência } \\
\text { à penetraçãa } \\
(\mathrm{MPa})\end{array}$ & $\begin{array}{c}\text { Densidade } \\
\left(\mathrm{Mg} \mathrm{m}^{-3}\right)\end{array}$ & $\begin{array}{c}\text { Produtividade } \\
\text { relativa }(\%)\end{array}$ \\
\hline $\mathrm{T}_{0}$ & 0,39 & 1,32 & $100,0 \mathrm{a}$ \\
$\mathrm{T}_{1}$ & 1,82 & 1,62 & $97,8 \mathrm{a}$ \\
$\mathrm{T}_{2}$ & 2,73 & 1,73 & $34,0 \mathrm{~b}$ \\
$\mathrm{~T}_{4}$ & 3,87 & 1,78 & $7,1 \mathrm{~b}$ \\
$\mathrm{~T}_{6}$ & 4,25 & 1,80 & $2,8 \mathrm{~b}$
\end{tabular}

${ }^{11} \mathrm{~T}_{0}, \mathrm{~T}_{1}, \mathrm{~T}_{2}, \mathrm{~T}_{4}, \mathrm{~T}_{6}$ correspondem a zero, uma, duas, quatro e seis passadas do trator, lado a lado, na superfície do solo, respectivamente. Resistência do solo à penetração determinada com conteúdo de água retida em capacidade de campo $(0,01 \mathrm{MPa})$ com composição granulométrica semelhante, pode ser atribuída, em parte, segundo Johnson et al. (1990), ao fato de que nem sempre a redução do crescimento vegetativo reflete menor produtividade da cultura.

Em solos compactados, menor crescimento radicular e menor produtividade das culturas ocorrem em razão do menor volume de solo explorado pelas raízes por causa da menor penetração, ramificação e espessamento (Guimarães \& Moreira, 2001), diminuindo sua eficiência na absorção de água e nutrientes (Hakansson \& Voorhees, 1998), além do fato de que a difusão de Pe K diminui à medida que a compactação aumenta (Novais \& Smyth, 1999).

A partir da Ds de $1,3 \mathrm{Mg} \mathrm{m}^{-3}$, o IHO é limitado na parte inferior pela RP, ou seja, apenas na condição de solo solto, a RP não foi o atributo redutor do IHO, e o limite superior foi o conteúdo de água em capacidade de campo (tensão de 0,01 MPa) (Figura 2). Tormena et al. (1998) verificaram que em solos tropicais o IHO foi limitado na parte superior pelo conteúdo de água na capacidade de campo e na parte inferior, pela resistência do solo à penetração. Neste sentido, Imhoff et al. (2000) propõem a utilização da curva de RP no monitoramento da qualidade física do solo e orientação de práticas de manejo do solo em pastagens.



Figura 2. Variação do conteúdo de água volumétrico $(\theta)$ com a densidade do solo, para os limites críticos da porosidade de aeração $\left(\theta_{\mathrm{PA}}, \mathbf{\Lambda}\right)$, capacidade de campo $\left(\theta_{\mathrm{CC}}, \Delta\right)$, resistência do solo à penetração de $2,0 \mathrm{MPa}\left(\theta_{\mathrm{RP}}, \bullet\right)$ e ponto de murcha permanente $\left(\theta_{\mathrm{PMP}}, \mathrm{O}\right)$ no Latossolo Vermelho distrófico. IHO: intervalo hídrico ótimo. Dsc: Densidade do solo crítica. A RP variou entre 0,05 e $18,90 \mathrm{MPa}$; a Ds de 1,11 a $1,85 \mathrm{Mg} \mathrm{m}^{-3}$; e o conteúdo de água volumétrica de $0,07 \mathrm{a} 0,37 \mathrm{~m}^{3} \mathrm{~m}^{-3}$. 
O IHO variou negativamente com decréscimo linear a partir da $\mathrm{Ds}$ de $1,3 \mathrm{Mg} \mathrm{m}^{-3}$ até o valor zero denominado de densidade do solo crítica (Dsc), valor de Ds a partir do qual as condições ao desenvolvimento radicular são acentuadamente restritivas. Silva et al. (1994) afirmam que a freqüência de valores de conteúdo de água fora do IHO depende da sua magnitude e da variabilidade do conteúdo de água do solo em função do clima.

A Dsc obtida no IHO foi de $1,63 \mathrm{Mg} \mathrm{m}^{-3}$, valor de Ds a partir do qual o crescimento radicular é restringido pela excessiva RP do solo, quando o conteúdo de água do solo corresponder à capacidade de campo (Figura 2). Se o conteúdo de água for inferior, a restrição ao crescimento radicular ocorre em valores inferiores de Ds, segundo o modelo do intervalo hídrico ótimo. Assim, em condições de campo, a produtividade das culturas deve ser reduzida em valores inferiores ou próximos a Ds de $1,63 \mathrm{Mg} \mathrm{m}^{-3}$, o que de fato ocorreu com o arroz. O valor de Ds a partir do qual a produtividade de arroz foi restringida foi de $1,62 \mathrm{Mg} \mathrm{m}^{-3}$, porém o conteúdo de água no campo não é sempre o conteúdo de água retida em capacidade de campo.

Até 70 dias após a semeadura o conteúdo de água no solo permaneceu poucos dias abaixo da capacidade de campo, normalmente atingida dois a três dias após a chuva (Reichardt, 1985). O crescimento radicular, porém, não cessou aos 70 dias, e após este período ocorreram estiagens (Figura 1). Assim, possivelmente, o crescimento de raízes foi restringido antes da Dsc de 1,63 $\mathrm{Mg} \mathrm{m}^{-3}$. Pabin et al. (1998) afirmam que muitas vezes ocorrem mudanças e redução no comprimento do sistema radicular, que mantém, satisfatoriamente, o suprimento de água e nutrientes para a parte aérea, não refletindo em menor produtividade, sendo a redução de $40 \%$ do crescimento radicular crítica à produtividade plantas. Isso justifica a redução da produtividade do arroz a partir da densidade do solo crítica.

O modelo do IHO indica que, em condições de conteúdo de água correspondente à capacidade de campo, o crescimento radicular é restringido a partir da densidade do solo crítica. Assim, como o conteúdo de água no solo foi inferior à capacidade de campo vários dias durante o desenvolvimento da cultura (Figura 1), a redução da produtividade do arroz de sequeiro na Ds correspondente a Dsc está de acordo com a proposição do modelo. Silva (2003), em Latossolo Vermelho, verificou que a cultura do feijão, em preparo reduzido do solo, permaneceu três dias a mais no IHO, comparado ao preparo convencional e plantio direto, contudo não proporcionou incrementos na produtividade.
A compactação do solo pelo tráfego da máquina foi mais pronunciada até a profundidade de $0,18 \mathrm{~m}$ (Tabela 1), conforme observado por Silva et al. (2000). Assim, foram calculadas as correlações entre a produtividade do arroz e a RP, Ds, porosidade total, macroporosidade e microporosidade, tendo-se encontrado os valores de $\mathrm{r}$ iguais a $-0,94,-0,85,0,79,-0,44 \mathrm{e}$ 0,75 , respectivamente. Apenas a RP apresentou correlação significativa. Taylor \& Gardner (1963) encontraram correlação de $-0,96$ entre a RP e crescimento radicular de algodão, superior à correlação de $-0,59$ com a densidade do solo.

Duas passagens de uma máquina de $11 \mathrm{Mg}$ no mesmo local, um dia após a chuva, foram suficientes para compactar o solo e reduzir em $66 \%$ a produtividade de arroz de sequeiro, em Latossolo Vermelho, destacando a importância e a necessidade de trafegar o solo mais seco, a fim de reduzir os custos de produção. Streck (2003) relata redução de 20 e $40 \%$ na produtividade da soja e do feijão, respectivamente, com duas passagens de uma máquina de 10 Mg no mesmo local em condições de conteúdo de água acima do ponto de friabilidade do Argisolo Vermelho-Amarelo de textura arenosa.

\section{Conclusões}

1. A compactação do solo a partir de um valor de resistência do solo à penetração de 1,82 $\mathrm{MPa}$ e de densidade do solo de $1,62 \mathrm{Mg} \mathrm{m}^{-3}$ reduz a produtividade do arroz de sequeiro no Latossolo Vermelho de textura média.

2. A determinação do intervalo hídrico ótimo é um parâmetro importante no monitoramento da compactação do solo e na prevenção de perdas de produtividade do arroz de sequeiro.

\section{Agradecimentos}

À Fapesp pela concessão de bolsa aos autores Amauri Nelson Beutler e Cassiano Garcia Roque; ao CNPq pela concessão de bolsa aos autores José Frederico Centurion e Alvaro Pires da Silva; à Capes pela concessão de bolsa ao autor Marcos Vieira Ferraz.

\section{Referências}

BEUTLER, A.N.; CENTURION, J.F.; SOUZA, Z.M.; SILVA, L.M. Utilização dos penetrômetros de impacto e de anel dinamométrico em Latossolos. Engenharia Agrícola, v.22, p.191-199, 2002. 
BUSSCHER, W.J. Adjustment of flat-tipped penetrometer resistance data to a common water content. Transactions of the ASAE v.3, p.519-524, 1990

CAMARGO, O.A.; ALLEONI, L.R.F. Compactação do solo e desenvolvimento das plantas. Piracicaba: Esalq, 1997. 132p.

EMBRAPA. Centro Nacional de Pesquisa de Solos (Rio de Janeiro, RJ). Manual de métodos de análise de solo. 2.ed. Rio de Janeiro, 1997. $212 p$

GENUCHTEN, M.T.A. van. A closed-form equation for predicting the hydraulic conductivity of unsaturated soils. Soil Science Society of America Journal, v.44, p.892-897, 1980

GUIMARÃES, C.M.; MOREIRA, J.A.A. Compactação do solo na cultura do arroz de terras altas. Pesquisa Agropecuária Brasileira, v.36, p. 703-707, 2001.

HAKANSSON, I.; VOORHEES, W.B. Soil compaction. In: LAL, R.; BLUM, W.H.; VALENTINE, C.; STEWARD, B.A. (Ed.) Methods for assessment of soil degradation. Boca Raton: CRS Press, 1998. p.167-179. (Advances in Soil Science).

HERRICK, J.E.; JONES, T.L. A dynamic cone penetrometer for measuring soil penetration resistance. Soil Science Society of America Journal, v.66, p.1320-1324, 2002.

HOAD, S.P.; RUSSEL, G; LUCAS, M.E.; BINGHAM, I.J. The management of wheat, barley, and oat root systems. Advances in Agronomy, v.74, p.195-254, 2001.

IMHOFF, S.; SILVA, A.P.; TORMENA, C.A. Aplicações da curva de resistência no controle da qualidade física de um solo sob pastagem Pesquisa Agropecuária Brasileira, v.35, p.1493-1500, 2000. JOHNSON, J.F.; VOORHEES, W.B.; NELSON, W.W.; RANDALL, G.W. Soybean growth and yield as affected by surface and subsoil compaction. Agronomy Journal, v.82, p.973-979, 1990.

MEROTTO, A.; MUNDSTOCK, C.M. Wheat root growth as affected by soil strength. Revista Brasileira de Ciência do Solo, v.23, p.197-202, 1999.

MIELNICZUK, J.; CARPENEDO, V.; PEDO, F. Desenvolvimento de raízes em solos compactados. Lavoura Arrozeira, v.38, p.42-43, 1985.

MOURA NETO, F.P. Desempenho de cultivares de arroz de terras altas sob plantio direto e convencional. 2001. 92p. Dissertação (Mestrado) - Universidade Federal de Larvas, Lavras. NOVAIS, R.F.; SMYTH, T.J. Fósforo em solo e planta em condições tropicais. Viçosa: UFV, 1999. 399p.

PABIN, J.; LIPIEC, J.; WODEK, S. Critical soil bulk density and strength for pea seedling root growth as related to other soil factors. Soil and Tillage Research, v.19, p.131-143, 1998

RAIJ, B. van; CANTARELLA, H.; QUAGGIO, J.A.; FURLANI A.M.C. Recomendação de adubação e calagem para o Estado d São Paulo. 2.ed. Campinas: Instituto Agronômico, 1996, 285p. (Boletim Técnico 100).
RAIJ, B. van; QUAGGIO, J.A.; CANTARELLA, H.; FERREIRA, M.E.; LOPES, A.S.; BATAGLIA, O.C. Análise química do solo para fins de fertilidade. Campinas: Fundação Cargill, 1987. 170p. RALISCH, R.; TAVARES FILHO, J. Compactação: uma preocupação pertinente. A Granja, v.646, p.55-57, 2002.

REICHARDT, K. Capacidade de campo. Revista Brasileira de Ciência do Solo, v.12, p.211-216, 1988.

REICHARDT, K. Processos de transferência no sistema solo planta - atmosfera. Piracicaba: Fundação Cargill, 1985. 445p.

ROSOLEM, C.A.; FERNANDEZ, E.M.; ANDREOTTI, M.; CRUSCIOL, C.A.C. Crescimento radicular de plântulas de milho afetado pela resistência do solo à penetração. Pesquisa Agropecuária Brasileira, v.34, p.821-828, 1999.

SILVA, A.P.; KAY, B.D.; PERFECT, E. Characterization of the least limiting water range. Soil Science Society of America Journal, v.58, p.1775-1781, 1994

SILVA, V.R. da. Propriedades físicas e hídricas em solos sob diferentes estados de compactação. 2003. 171p. Tese (Doutorado) - Universidade Federal de Santa Maria, Santa Maria.

SILVA, V.R. da; REINERT, D.J.; REICHERT, J.M. Resistência mecânica do solo à penetração influenciada pelo tráfego de uma colhedora em dois sistemas de manejo do solo. Ciência Rural, v.30, p.795-801, 2000.

SMITH, C.W.; JOHNSTON, M.A.; LORENTZ, S. The effect of soil compaction and soil physical properties on the mechanical resistance of South African forestry soils. Geoderma, v.78, p.93111, 1997.

STONE, L.F.; GUIMARÃES, C.M.; MOREIRA, J.A.A. Compactação do solo na cultura do feijoeiro - I: efeitos nas propriedades físico-hídricas do solo. Revista Brasileira de Engenharia Agrícola e Ambiental, v.6, p.207-212, 2002.

STRECK, C.A. Compactação do solo e seus efeitos no desenvolvimento radicular e produtividade da cultura do feijoeiro e da soja. 2003. 83p. Dissertação (Mestrado) Universidade Federal de Santa Maria, Santa Maria.

TARDIEU, F. Growth and functioning of roots and to root systems subjected to soil compaction: towards a system with multiple signaling. Soil and Tillage Research, v.30, p.217-243, 1994.

TAVARES FILHO, J.; BARBOSA, G.M.C.; GUIMARÃES, M.F.; FONSECA, I.C.B. Resistência do solo à penetração e desenvolvimento do sistema radicular do milho (Zea mays) sob diferentes sistemas de manejo em um Latossolo Roxo. Revista Brasileira de Ciência do Solo, v.25, p.725-730, 2001

TAYLOR, H.M.; GARDNER, H. Penetration of cotton seedling taproots as influenced by bulk density, moisture content, and strength of soil. Soil Science, v.96, p.153-156, 1963.

TORMENA, C.A.; SILVA, A.P.; LIBARDI, P.L. Caracterização do intervalo hídrico ótimo de um Latossolo Roxo sob plantio direto. Revista Brasileira de Ciência do Solo, v.22, p.573-581, 1998.

Recebido em 23 de dezembro de 2003 e aprovado em 22 de março de 2004 